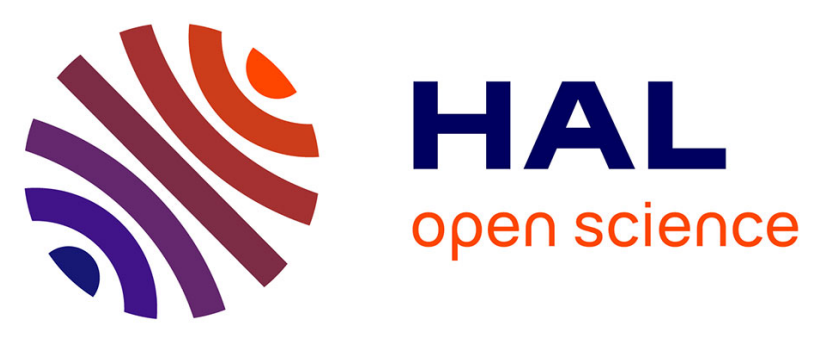

\title{
Measurement of acoustic streaming in a closed-loop traveling wave resonator using laser Doppler velocimetry
}

Cyril Desjouy, Guillaume Penelet, Pierrick Lotton, James Blondeau

\section{To cite this version:}

Cyril Desjouy, Guillaume Penelet, Pierrick Lotton, James Blondeau. Measurement of acoustic streaming in a closed-loop traveling wave resonator using laser Doppler velocimetry. Journal of the Acoustical Society of America, 2009, 126 (5), pp.2176-2183. 10.1121/1.3238162 . hal-02057435

\section{HAL Id: hal-02057435 \\ https://hal-univ-lemans.archives-ouvertes.fr/hal-02057435}

Submitted on 5 Mar 2019

HAL is a multi-disciplinary open access archive for the deposit and dissemination of scientific research documents, whether they are published or not. The documents may come from teaching and research institutions in France or abroad, or from public or private research centers.
L'archive ouverte pluridisciplinaire HAL, est destinée au dépôt et à la diffusion de documents scientifiques de niveau recherche, publiés ou non, émanant des établissements d'enseignement et de recherche français ou étrangers, des laboratoires publics ou privés. 


\title{
Measurement of acoustic streaming in a closed-loop traveling wave resonator using laser Doppler velocimetry
}

\author{
Cyril Desjouy, ${ }^{\text {a) }}$ Guillaume Penelet, Pierrick Lotton, and James Blondeau \\ Laboratoire d'Acoustique de l'Université du Maine, UMR CNRS 6613, Avenida Olivier Messiaen, \\ 72085 Le Mans Cedex 9, France
}

(Received 25 March 2009; revised 24 August 2009; accepted 25 August 2009)

\begin{abstract}
This paper deals with the measurement of acoustic particle velocity and acoustic streaming velocity in a closed-loop waveguide in which a resonant traveling acoustic wave is sustained by two loudspeakers appropriately controlled in phase and amplitude. An analytical model of the acoustic field and a theoretical estimate of the acoustic streaming are presented. The measurement of acoustic and acoustic streaming velocities is performed using laser Doppler velocimetry. The experimental results obtained show that the curvature of the resonator impacts the acoustic velocity and the profile of acoustic streaming. The quadratic dependence of the acoustic streaming velocity on the acoustic pressure amplitude is verified and the measured cross-sectional average streaming velocity is in good agreement with the value predicted by the theoretical estimate.
\end{abstract}

(C) 2009 Acoustical Society of America. [DOI: 10.1121/1.3238162]

PACS number(s): 43.25.Nm [OAS]

Pages: $2176-2183$

\section{INTRODUCTION}

Acoustic streaming is a net mean flow which is generated by sound. This nonlinear effect is known for more than a century, ${ }^{1}$ and there is renewed interest in studying this effect because it may be used, for instance, to enhance heat transfer, ${ }^{2}$ to generate fluid motion in microfluidic devices, ${ }^{3,4}$ or to drive ultrasonic motors. ${ }^{5,6}$ Acoustic streaming can also disturb the operation of thermoacoustic engines ${ }^{7}$ because it is responsible for generally unwanted heat convection within the device. Even though the influence of acoustic streaming on the efficiency of thermoacoustic engines has been already studied, ${ }^{8,9}$ it is still poorly understood. Thus, its effects on the operation of engines are usually almost empirically controlled, using jet pumps ${ }^{10,11}$ and/or tapered tubes. ${ }^{12}$ The characterization of acoustic streaming in thermoacoustic engines is indeed a difficult task due to large temperature gradients and complicated shapes of the different elements of the engine.

Efforts have been devoted to the theoretical description of acoustic streaming in acoustic resonators ${ }^{13-16}$ and in thermoacoustic devices. ${ }^{17-21}$ The precise measurement of acoustic streaming velocity has also been recently achieved by Thompson et al. ${ }^{22,23}$ and Moreau et al. ${ }^{24}$ using laser Doppler velocimetry (LDV) and appropriate signal processing. Thompson et al. measured the Lagrangian outer (i.e., outside acoustic boundary layers) streaming velocity in a standing wave and they studied the influences of the temperature gradients and the fluid inertia on acoustic streaming. They notably demonstrated that the dependence of viscosity on temperature impacts the acoustic streaming velocity as predicted by Rott. ${ }^{13}$ They also showed that small temperature gradients induce significant discrepancies between the observed velocities and any available theoretical results. Moreau et al.

\footnotetext{
a) Author to whom correspondence should be addressed. Electronic mail: cyril.desjouy@univ-lemans.fr
}

measured both inner/outer and slow/fast streaming velocities using LDV and they focused attention to the spatial distribution of acoustic streaming near the walls (inside the acoustic boundary layers). It is, however, remarkable that, while recent developments of thermoacoustic engines make use of a closed-loop path to increase the efficiency, ${ }^{10,11,25}$ most of the studies mentioned above are concerned with standing wave devices. Just a few studies deal with traveling wave devices for which the existence of a closed-loop notably allows the streaming flow to be nonzero across the section of the resonator.

Thus, this paper aims at contributing to a better understanding of acoustic streaming behavior in closed-loop acoustic resonators. The device studied in this paper is not a thermoacoustic engine. It consists of an annular waveguide in which the acoustic field is a resonant acoustic traveling wave generated and controlled by two loudspeakers (Fig. 1). Laser Doppler velocimetry (LDV) measurements of acoustic particle velocity and acoustic streaming velocity are performed in this study, and the results are compared to simplified theoretical models. It should be mentioned that, though the idea of using a closed-loop path to generate a guided traveling wave is not new (see, for instance, Refs. 26-28), we did not find in the literature any report of experiments in the device which is described below.

In Sec. II, the experimental apparatus is briefly described. In Sec. III, an analytical modeling of the acoustic field and a theoretical estimate of the acoustic streaming velocity are presented. The LDV measurement of acoustic particle velocity and acoustic streaming velocity are presented and discussed in Sec. IV.

\section{EXPERIMENTAL APPARATUS}

A schematic representation of the experimental device is shown in Fig. 1(a). It consists of an annular resonator of unwrapped length $L=2.12 \mathrm{~m}$ and with a square cross-section 
(a)
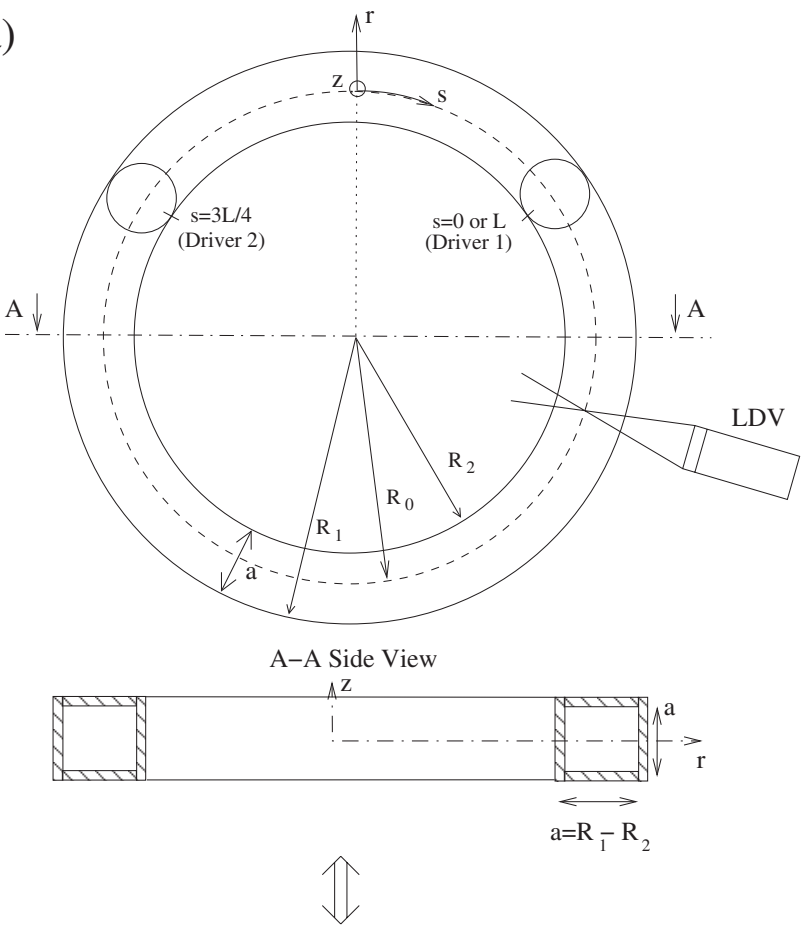

(b)

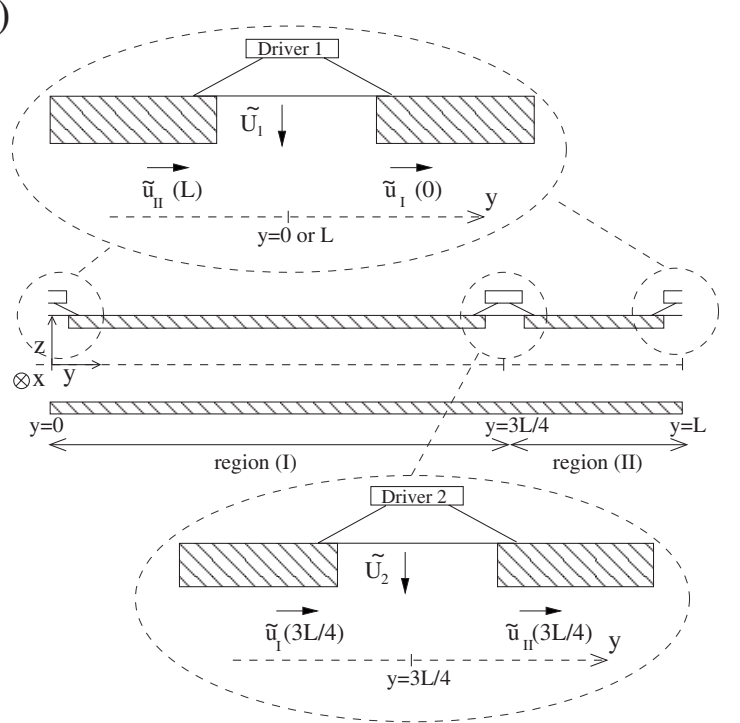

FIG. 1. (a) Schematic representations of the experimental device (top view and side view). (b) Unwrapped schematic representation of the experimental device, the cylindrical system of coordinates $(r, \theta, z)$ [or $(r, s, z)$ with $s$ $=\theta L / 2 \pi]$ being replaced by the Cartesian coordinates $(x, y, z)$. The drivers localized at $s$ or $y=0$ (or $L$ ) and $s$ or $y=3 L / 4$ are represented with details on the volume velocities and their orientations. Simultaneous measurements of acoustic and acoustic streaming velocities in the longitudinal direction ( $s$ or y) are performed using a commercial laser Doppler velocimeter (Dantec Dynamics, Flowlite 1D).

$S_{W}=a \times a=7.5 \times 7.5 \mathrm{~cm}^{2}$. The waveguide, which is filled with air at atmospheric pressure and at room temperature $\left(20^{\circ} \mathrm{C}\right)$, is made of Plexiglass ${ }^{\mathrm{TM}}$ to allow LDV measurements of acoustic particle velocity and acoustic streaming velocity. Two electrodynamic drivers (loudspeakers Audax PR170MO), set at positions $s=0$ and $s=3 L / 4$, and coupled to the waveguide through a circular hole of diameter $a$, allow to generate an acoustic wave inside the resonator. Particular attention is paid to the accurate control of the drivers: a laser
Doppler vibrometer (Polytec OFV 300) is used to measure both amplitude and phase of the oscillating velocities at the center of the loudspeaker membranes. Three microphones (Brüel \& Kjær 4136) are flush-mounted along the resonator (located at $s=0.57 \mathrm{~m}, s=0.66 \mathrm{~m}$, and $s=0.75 \mathrm{~m}$ from the driver 1 set at $s=0$ ).

The specificity of the experimental device is that, under some circumstances, it is possible to sustain a resonant rotating wave $\mathrm{e}^{29}$ which has the characteristics of a traveling wave in terms of spatial distribution of the acoustic field (and in terms of phase shift between acoustic pressure and velocity fluctuations). More precisely, it has been demonstrated (see, for instance, Ref. 26) that if the drivers are separated by a distance of $L / 4$, if each driver is sustaining sound at a frequency $f$ which corresponds to the first natural mode of the air column $\left(f=c_{0} / L \approx 161 \mathrm{~Hz}\right.$, where $c_{0} \approx 342 \mathrm{~m} \mathrm{~s}^{-1}$ is the adiabatic speed of sound), and if the drivers displacements are equal in amplitude but $\pi / 2$ out of phase, then the resulting acoustic wave propagating into the waveguide behaves like a resonant traveling wave (provided that the dissipation of acoustic energy in the waveguide is negligible). This is due to the fact that each driver generates two counterpropagating acoustic waves in the resonator, two of them propagating in one direction being coherently additive (in direction $+\mathbf{s}$, for instance, if the driver 2 has a phase delay of $-\pi / 2$ compared to driver 1 ) while the two other waves canceling out.

\section{ANALYTICAL DESCRIPTION OF THE ACOUSTIC FIELD AND OF THE ACOUSTIC STREAMING}

\section{A. Acoustic volume velocity}

A simplified analytical description of the acoustic field is presented in this section. It is assumed here that the curvature of the resonator has no influence on the propagation of the acoustic wave inside the resonator, so that the closed-loop waveguide [Fig. 1(a)] is assumed to be equivalent to the unwrapped waveguide [Fig. 1(b)], the "closed-loop" property of the resonator being taken into account by ensuring continuity of acoustic pressure and acoustic velocity between both ends of the unwrapped resonator. Assuming that a plane acoustic wave takes place in the waveguide, the acoustic pressure inside the resonator can be written $p(y, t)$ $=\mathfrak{R}\left(\widetilde{p}(y) e^{-i \omega t}\right)$, where $\mathfrak{R}$ denotes taking the real part of the complex argument and where ${ }^{\sim}$ denotes the complex amplitude. In the following, the axial component of the acoustic particle velocity is denoted $v_{y}(x, y, z, t)=\mathfrak{R}\left(\widetilde{v}_{y}(x, y, z) e^{-i \omega t}\right)$. The two loudspeaker membranes provide harmonic motion at frequency $f=\omega /(2 \pi)=c_{0} / L$. The complex amplitudes of acoustic volume velocities generated by the loudspeaker 1 , located at $y=0$, and the loudspeaker 2, located at $y=3 L / 4$, are denoted by $\widetilde{U}_{1}$ and $\widetilde{U}_{2}$, respectively. Two regions, denoted by I and II [Fig. 1(b)], split the closed-loop resonator. The complex amplitude $\widetilde{p}_{\text {I,II }}$ of acoustic pressure in each region is written as follows:

$$
\widetilde{p}_{\mathrm{I}, \mathrm{II}}(y)=\widetilde{p}_{\mathrm{I}, \mathrm{II}}^{+}\left(y_{\mathrm{I}, \mathrm{II}}\right) e^{+i k_{w}\left(y-y_{\mathrm{I}, \mathrm{II}}\right)}+\widetilde{p}_{\mathrm{I}, \mathrm{II}}\left(y_{\mathrm{I}, \mathrm{II}}\right) e^{-i k_{w}\left(y-y_{\mathrm{I}, \mathrm{II}}\right)},
$$

where $y_{\mathrm{I}, \mathrm{II}}$ denote any abscissa in regions I and II, respectively, and where 


$$
k_{w}=k_{0} \sqrt{1+\frac{f_{\nu}+(\gamma-1) f_{\kappa}}{1-f_{\nu}}}
$$

is the complex wave number which accounts for viscous and thermal dissipation in the vicinity of the resonator's walls (the functions $f_{\nu}$ and $f_{\kappa}$ characterize the viscous and thermal coupling between the wall of the resonator and the oscillating fluid, ${ }^{7} k_{0}=\omega / c_{0}$, and $\gamma$ is the specific heat ratio). The complex amplitudes of the acoustic volume velocities $\widetilde{u}_{\mathrm{I}, \mathrm{II}}$ $=S_{W} \cdot\left\langle\widetilde{v}_{y_{\mathrm{III}}}(x, y, z)\right\rangle$, where $\langle\cdots\rangle$ is used to denote the crosssectional spatial average, are obtained from the acoustic pressure as follows: ${ }^{7}$

$$
\widetilde{u}_{\mathrm{I}, \mathrm{II}}(y)=\frac{S_{W}\left(1-f_{\nu}\right)}{i \omega \rho_{0}} \frac{\partial \widetilde{p}_{\mathrm{I}, \mathrm{II}}}{\partial y},
$$

where $\rho_{0}$ stands for the mean density of fluid. Setting $y_{\mathrm{I}}=0$ and $y_{\mathrm{II}}=3 \mathrm{~L} / 4$, and assuming that, at the loudspeaker locations, there is continuity of acoustic pressure $\left[\widetilde{p}_{\mathrm{I}}(0)=\widetilde{p}_{\mathrm{II}}(L)\right.$ and $\left.\widetilde{p}_{\mathrm{I}}(3 L / 4)=\widetilde{p}_{\mathrm{II}}(3 L / 4)\right]$ and continuity of acoustic volume velocity $\left[\widetilde{u}_{\mathrm{II}}(L)+\widetilde{U}_{1}=\widetilde{u}_{\mathrm{I}}(0)\right.$ and $\left.\tilde{u}_{\mathrm{I}}(3 L / 4)+\widetilde{U}_{2}=\widetilde{u}_{\mathrm{II}}(3 L / 4)\right]$, the constants $\widetilde{p}_{\mathrm{I}}^{ \pm}\left(y_{\mathrm{I}}\right)$ and $\tilde{p}_{\mathrm{II}}^{ \pm}\left(y_{\mathrm{II}}\right)$ can be expressed as functions of $\tilde{U}_{1,2}$. In particular, if $\widetilde{U}_{2}=\widetilde{U}_{1} e^{i \phi}$, this leads to the following expressions for the acoustic volume velocity in the entire resonator:

$$
\begin{aligned}
\tilde{u}_{\mathrm{I}}= & \frac{i \widetilde{U}_{\mathrm{I}}}{4 \sin \left(k_{w} L / 2\right)}\left[\left(e^{-i\left(k_{w}(L / 4)-\phi\right)}+e^{-i k_{w}(L / 2)}\right) e^{i k_{w} y}\right. \\
& \left.-\left(e^{i\left(k_{w}(L / 4)+\phi\right)}+e^{i k_{w}(L / 2)}\right) e^{-i k_{w} y}\right], \\
\tilde{u}_{\mathrm{II}}= & \frac{i \widetilde{U}_{1}}{4 \sin \left(k_{w} L / 2\right)}\left[\left(e^{i k_{w}(L / 4)}+e^{-i\left(k_{w}(L / 2)-\phi\right)}\right) e^{i k_{w}(y-(3 L / 4))}\right. \\
& \left.-\left(e^{-i k_{w}(L / 4)}+e^{i\left(k_{w}(L / 2)+\phi\right)}\right) e^{-i k_{w}(y-(3 L / 4))}\right] .
\end{aligned}
$$

Moreover, notifying that for the given angular frequency $\omega=2 \pi c_{0} / L$, the viscous and thermal boundary layer thicknesses $\delta_{\nu}=\sqrt{2 \nu / \omega}$ and $\delta_{\kappa}=\sqrt{2 \kappa / \omega}(\nu$ and $\kappa$ being kinematic viscosity and thermal diffusivity of fluid, respectively) are small compared to the hydraulic radius $a$ of the resonator, the viscous and thermal functions $f_{\nu, \kappa}$ can be approximated by ${ }^{30}$

$$
f_{\nu, \kappa} \stackrel{\delta_{\nu, \kappa} \ll a}{\approx}(1+i) \frac{\delta_{\nu, \kappa}}{a} .
$$

Due to this, the complex wavenumber is approximated by $k_{w} \approx k_{0}(1+\epsilon)+i k_{0} \epsilon$, where $\epsilon$ is a small parameter given by $\epsilon=\delta_{\kappa}[(\gamma-1)+\sqrt{\sigma}] /(2 a) \ll 1$ and where $\sigma=\nu / \kappa$ denotes the Prandtl number. Tuning adequately the relative phase shift $\phi$ between the membranes displacements of the loudspeakers allows to generate a given kind of acoustic wave. In particular, if $\phi=-\pi / 2$, the approximate expressions of the acoustic volume velocities $\widetilde{u}_{\mathrm{I}, \mathrm{II}}$ obtained by expanding Eqs. (4) and (5) over the small parameter $\epsilon$, can be written as follows:

$$
\widetilde{u}_{\mathrm{I}}(y)=\frac{-\widetilde{U}_{1}}{4 \epsilon \pi(1-i)}\left\{-2 e^{i k_{0} y}-\epsilon g_{1}(y)+O\left(\epsilon^{2}\right)\right\},
$$

$$
\begin{aligned}
\tilde{u}_{\mathrm{II}}(y)= & \frac{-\tilde{U}_{1}}{4 \epsilon \pi(1-i)}\left\{2 i e^{i k_{0}(y-(3 L / 4))}+\epsilon g_{2}\left(y-\frac{3 L}{4}\right)\right. \\
& \left.+O\left(\epsilon^{2}\right)\right\},
\end{aligned}
$$

where

$$
\begin{aligned}
& g_{1}(\xi)=\pi(1-i)\left[\cos \left(k_{0} \xi\right)+\left(1-\frac{4 \xi}{L}\right) e^{i k_{0} \xi}\right], \\
& g_{2}(\xi)=\pi(1+i)\left[3 \cos \left(k_{0} \xi\right)-\left(1+\frac{4 \xi}{L}\right) e^{i k_{0} \xi}\right] .
\end{aligned}
$$

This result clearly shows that under the conditions mentioned above, the acoustic volume velocity can be separated into two components: the first one (of order of magnitude $1 / \epsilon)$ corresponds to a resonant acoustic wave traveling along the direction $+y$ and the second one (of order 1) corresponds to some spatial variations in acoustic amplitude due to the viscous and thermal boundary layers effects near the walls. So, as long as the parameter $\epsilon$ can be considered as small, the present device can be used as a traveling wave resonator.

\section{B. Cross-sectional average streaming velocity}

When a high level resonant acoustic traveling wave is sustained into the resonator, an acoustic streaming flow is generated by nonlinear effects, which presents a nonzero cross-sectional average value due to the closed-loop geometry. An approximate expression of this streaming velocity can be obtained using a successive approximation approach, assuming (1) that the boundary layer approximation is valid, (2) that the effect of the curvature of the resonator is neglected, and (3) that the dependence of kinematic viscosity $\nu$ of fluid on temperature $T_{0}$ is taken into account (namely, ${ }^{13}$ $\nu \propto T_{0}^{\beta}$, with $\beta=0.73$ ).

In the following, the subscript $m$ is used to denote the second order in magnitude mean flow generated by acoustic streaming. In order to compare the results of measurements with the theoretical results, the cross-sectional average value of streaming velocity $\left\langle v_{y m}(y)\right\rangle$ is expressed as a function of acoustic pressure amplitude when the waveguide has a circular cross-section of diameter $a$. The details of calculation, which are based on previous works, ${ }^{18}$ are given in Appendix. The average value along the closed-loop waveguide $1 / L \oint\left\langle v_{y m}\right\rangle \cdot d y$ of the cross-sectional average streaming velocity $\left\langle v_{y m}\right\rangle$ is found to be proportional to acoustic intensity as follows:

$$
\frac{1}{L} \oint\left\langle v_{y m}\right\rangle \cdot d y=\alpha p_{\mathrm{rms}}^{2},
$$

where $p_{\text {rms }}$ is the root-mean-square of the acoustic pressure amplitude inside the waveguide, and where the parameter $\alpha \approx-1.8 \times 10^{-7} \mathrm{~m} \mathrm{~s}^{-1} \mathrm{~Pa}^{-2}$. It should be mentioned that acoustic streaming velocity is not constant along the waveguide, but the predicted variations in $\left\langle v_{y m}\right\rangle$ are lower than $0.5 \%$ of its average value. It is also interesting to note that the direction of acoustic streaming is opposite to the direction of the traveling wave. 

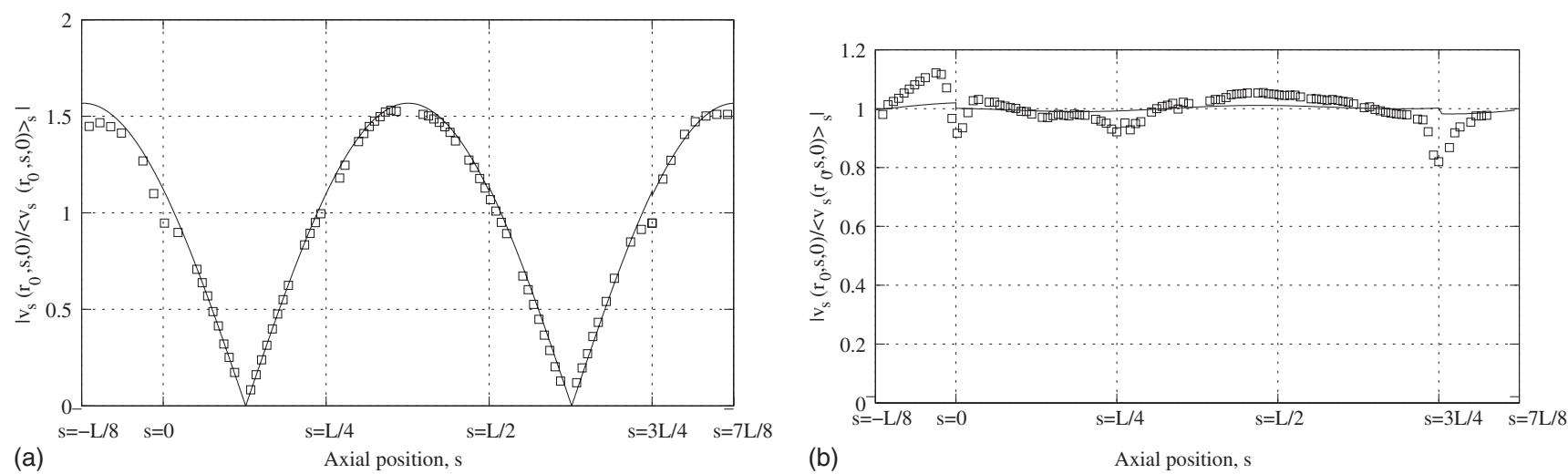

(b)

Axial position,

FIG. 2. Normalized distributions of the axial velocity fields $\left|v_{s}\left(R_{0}, s, 0\right) /\left\langle v_{s}\left(R_{0}, s, 0\right)\right\rangle_{s}\right|$ as function of the axial position $s$ : squares correspond to the experiment and full lines correspond to the theory. (a) $\phi=0$; (b) $\phi=-\pi / 2$.

\section{MEASUREMENT PROCEDURE}

\section{A. Hardware and signal processing}

For all the experimental results which are presented in the following, the absolute values of the amplitudes of the displacements of the loudspeaker membranes are equal $\left(\left|\tilde{U}_{1}\right|=\left|\tilde{U}_{2}\right|\right)$, and the operating frequency $f \approx\left(2 \pi c_{0}\right) / L$ $\approx 161 \mathrm{~Hz}$ corresponds to the frequency of the first natural acoustic mode of the air column. We have verified experimentally that this frequency also corresponds to a resonance of the complete device (the presence of the loudspeakers do not affect the acoustic resonance of the closed-loop waveguide). A commercial single component laser Doppler velocimeter (Dantec Dynamics, $158.4 \mathrm{~mm}$ standard front lens) is used to measure velocity field. The dimensions of the fringe volume are of about $50 \mu \mathrm{m}$ in the direction of measurement (along the $s$-coordinate) by $410 \mu \mathrm{m}$ wide (along the $r$-coordinate) and $50 \mu \mathrm{m}$ high (along the $z$-coordinate). The LDV probe can move in the transverse direction $r$ [or $x$ if the unwrapped geometry of Fig. 1(b) is considered]. The waveguide can rotate around its center, allowing to make measurements along the axial direction $s$ (or $y$ ). The signal processing which is used here is similar to that used by Moreau et $a .^{24}$ The signal is captured by a photomultiplier and is analyzed by the BSA system (Dantec Dynamics 57N20-BSA burst spectrum analyzer). Acquisition are performed over 40000 samples in order to reach convergence of the measurement results. Then, given a phase reference, a postprocessing algorithm allows to bring back all samples on a single acoustic period. A least squares method is used to estimate the Eulerian particle velocity. The average value (over an acoustic period) of the least squares fit of the signal corresponds to the streaming velocity $v_{s m}$ (or $v_{y m}$ in case of unwrapped waveguide), while the oscillating component corresponds to the acoustic particle velocity $v_{s}(t)\left[\right.$ or $\left.v_{y}(t)\right]$. The measurement accuracy is provided by the signal to noise ratio (SNR) defined as ${ }^{31} \mathrm{SNR}=10 \log \left(V_{s}^{2} / 2 \mathcal{V}[n(t)]\right)$, where $V_{s}$ denotes the peak amplitude of the acoustic particle velocity $v_{s}(t)$ and where $\mathcal{V}[n(t)]$ is the variance of the noise $n(t)$ (which is the difference between measured and estimated acoustic velocities). This SNR is used to calculate the minimal errors attributed to the data acquisition and the signal processing system, and in the following, LDV measurements are considered as valid for a SNR higher than $20 \mathrm{~dB}$. However, it is worth noting that there are additional uncertainties which are due to the experimental device itself. There are indeed some parameters like the evolution of room temperature or the accuracy of the probe positioning, which we tried to monitor with care. These parameters are not taken into account in the definition of the SNR, but may impact the accuracy of the measurements significantly. During measurements, we took care that the variations in room temperature do not exceed $1 \mathrm{~K}$, and we considered that the accuracy of probe positioning is lower than $1 \mathrm{~mm}$.

It is also worth noting that, in our device, the cascade process of higher harmonics generation should be considered because it distorts the waveform and because harmonics generation may contribute to the generation of acoustic streaming (this contribution is neglected in Sec. III B). However, for the largest amplitude of acoustic velocities used $(1.9 \mathrm{~m} / \mathrm{s})$, the amplitude of the second harmonic of particle velocity does not exceed $5.5 \%$ of the amplitude of the first harmonic. The assumption of a monofrequency acoustic field is thus expected to be valid because the magnitude of the acoustic streaming generated by this second-harmonic is expected to be less than $0.3 \%$ of the magnitude of the streaming generated by the first harmonic.

\section{B. Acoustic particle velocity measurements}

The spatial distribution along the centerline of the annular experimental resonator $\left[r=R_{0}, s, z=0\right.$, see Fig. 1(a) $]$ of the acoustic particle velocity is presented in Figs. 2(a) and 2(b) for a phase shift $\phi$ between the membrane displacements of the loudspeakers of 0 and $-\pi / 2$, respectively. More precisely, the normalized distributions of the axial velocity fields $\left|v_{s}\left(R_{0}, s, 0\right) /\left\langle v_{s}\left(R_{0}, s, 0\right)\right\rangle_{s}\right|$, where $\left\langle v_{s}\left(R_{0}, s, 0\right)\right\rangle_{s}$ denotes the average amplitude of acoustic velocity along the $s$-coordinate, are presented: full lines show the predicted spatial distribution [Eqs. (7) and (8)] and squares show the measurement results. The experimental and theoretical results are in good agreement. When $\phi=0$ [Fig. 2(a)], the spatial distribution of the acoustic field in the resonator is the one of a standing wave. When $\phi=-\pi / 2$ [Fig. 2(b)], the results show 


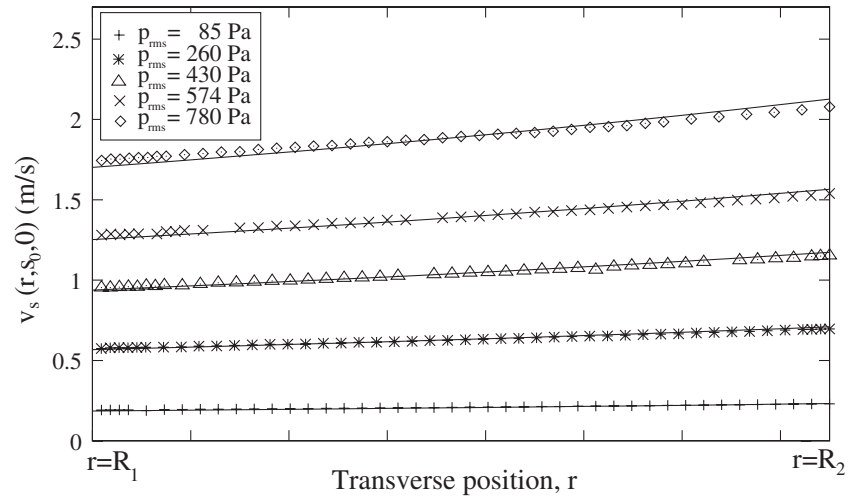

FIG. 3. Spatial distribution of the amplitude of the axial acoustic velocity $v_{s}\left(r, s_{0}, 0\right)$, measured by LDV at position $s_{0}=0.72 \mathrm{~m} \approx 3 L / 8$ for different amplitudes of acoustic pressure, with respect to the $r$-coordinate. Continuous lines correspond to the calculated distribution [Eq. (12)].

that the measured amplitude of the acoustic particle velocity (squares: $\square$ ) is nearly constant along the $s$-coordinate, as predicted by the theory (full line).

In Fig. 3, the measured spatial distribution of acoustic velocity with respect to coordinate $r$ is shown for different amplitudes of acoustic pressure. The phase shift between the two drivers is set to $\phi=-\pi / 2$. The LDV measurements are performed at position $s_{0}=0.72 \mathrm{~m} \approx 3 L / 8$. It was technically impossible here to measure this acoustic particle velocity inside the thermo-viscous boundary layers, so that the spatial distribution in Fig. 3 does not show that the particle velocity vanishes near the walls of the resonator, at positions $r=R_{1}$ and $r=R_{2}$. It appears clearly that the particle velocity is not uniform across a section of the waveguide. This effect is due to the curvature of the resonator, and an estimate of the actual velocity distribution can be obtained by assuming that, in the closed-loop resonator of Fig. 1(a), the pressure wave is a plane wave traveling in the $(+s)$ direction without dissipation $\left[\tilde{p}(r, s, z)=\tilde{p}(s)=\tilde{p}\left(s_{0}\right) e^{i k_{0}\left(s-s_{0}\right)}\right]$ so that, using Euler's equation in the cylindrical system of coordinates $(r, s, z)$, the complex amplitude of the axial acoustic particle velocity

$$
\tilde{v}_{s}\left(r, s_{0}, z\right)=\frac{1}{i \rho_{0} \omega r} \frac{\partial \widetilde{p}\left(s_{0}\right)}{\partial s}
$$

is proportional to $(1 / r) \partial_{s} \tilde{p}$. The corresponding transverse distribution of the acoustic particle velocity is also presented in Fig. 3 (continuous lines) and it is in good agreement with the results of measurements.

\section{Outer acoustic streaming velocity measurements}

Acoustic streaming velocity measurement by LDV is a tricky procedure in which several parameters such as the number of seeding particles getting through the measurement volume and their influence on the mean density of fluid, the temperature, and the static pressure variations impact the experimental results. The most annoying difficulty that we have encountered is the necessity to wait for quite a long time after the introduction of seeding particles (wood smoke) before to proceed to reliable acoustic streaming velocity measurements. Indeed, while the measured acoustic particle velocity converges to a constant value only several seconds after the introduction of seeding particles, the streaming velocity reaches its steady-state value after approximately 30 min, the initial streaming velocity value being one order of magnitude higher than its steady-state value. It should be mentioned that such an effect has already been reported by Thompson et $a l^{23}$ and confirmed by Moreau et $a .^{24}$ The most plausible reason which can explain this effect is the one invoked by Thompson et al., who have clearly demonstrated that the thermal boundary condition imposed on the walls of the resonator has a strong influence on both the characteristic time necessary to reach the steady-state acoustic streaming velocity and on the amplitude of the steady-state acoustic streaming velocity. In the present experiments, no thermal conditions are imposed on the resonator (this is referred to as "uncontrolled" boundary condition by Thompson et al.). The acoustic streaming velocity reaches its steady-state value within $27 \mathrm{~min}$ (the time required for the acoustic streaming velocity to reach $95 \%$ of its steady-state value), which is twice longer than the time of 14 min reported by Thompson et al. In the present device, this long time delay probably corresponds to the time for stabilization of a heterogeneous temperature distribution, which is due to various heat sources (notably the loudspeakers). Actually, this thermal equilibrium time is controlled by the convective heat transport due to the acoustic streaming which itself is controlled by the temperature distribution. In practice, this means that after each introduction of seeding particles into the waveguide, LDV measurements cannot begin before this 27 min time delay, and have to be stopped after approximately $1 \mathrm{~h}$ due to the gradual decrease in the number of seeding particles getting through the measurement volume, with subsequent decrease in the signal to noise ratio.

The measurement of the spatial distribution of the outer ("outer" meaning far from the boundary layers) acoustic streaming velocity $v_{s m}\left(R_{0}, s, 0\right)$ along the centerline is presented in Fig. 4 for both $\phi=0$ and $\phi=-\pi / 2$. It is important to note that it was not possible to prevent from annoying effects due to the loudspeakers in this device. This was not the case in the devices studied by Thompson et al. ${ }^{22}$ and Moreau et al.: ${ }^{24}$ Thompson et al. took care to proceed to the measurements far from the acoustic sources (approximately $1 \mathrm{~m}$ ) while Moreau et al. took care to design their waveguide in such a way that separation effects due to the geometrical singularities in the vicinity of the loudspeakers are minimized. In the present device, the disturbances due to the loudspeakers impact the measurement of acoustic streaming along almost half the length of the resonator (approximately from $s \approx 5 L / 8$ to $s \approx L / 8$ ). However, far away from the sources, after a stabilization distance of about $40 \mathrm{~cm}$, we consider that the observed streaming is the acoustic streaming itself (i.e., the streaming resulting from nonlinear acoustic effects in the vicinity of the resonator walls). In Fig. 4, both the acoustic particle velocity magnitude [Fig. 4(a)] and the acoustic streaming velocity magnitude [Fig. 4(b)] are represented along the waveguide. No matter how the phase shift between the loudspeakers is adjusted $(\phi=0$ or $\phi=$ $-\pi / 2$ ), the spatial zone where the measurements of acoustic streaming may be considered as reliable extends from $s$ $\approx L / 8$ to $s \approx 5 L / 8$. In this region, and in the case when a 

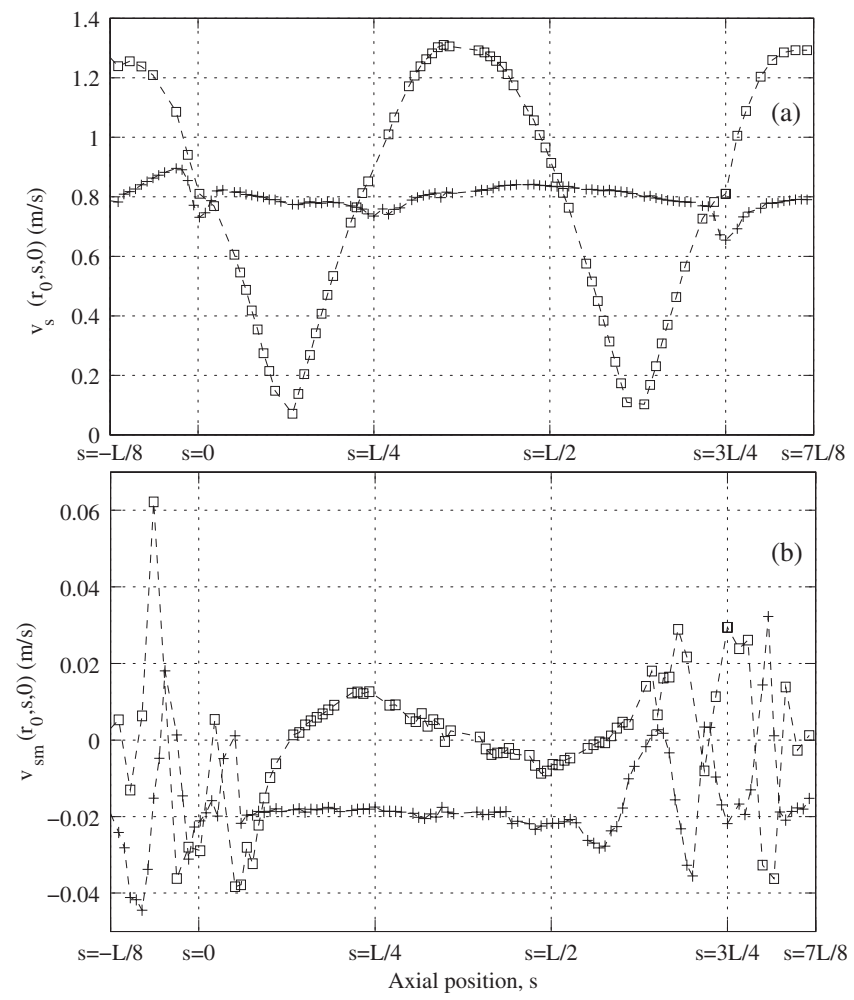

FIG. 4. (a) Spatial distribution of the amplitude of the axial acoustic velocity $v_{s}\left(R_{0}, s, 0\right)$ and (b) of the outer acoustic streaming velocity $v_{s m}\left(R_{0}, s, 0\right)$ with respect to the axial position $s$, measured by LDV for a phase shift $\phi$ $=-\pi / 2($ crosses: $\times)$ and $\phi=0$ (squares: $\square)$. In both cases $(\phi=0$ and $\phi$ $=-\pi / 2)$, the root-mean-square amplitude of the electric voltage applied to the loudspeakers is $1.8 \mathrm{~V}$.

standing wave is excited by the loudspeakers $(\phi=0$, squares: $\square$ ), it is interesting to note from the simultaneous observations of acoustic and acoustic streaming velocities that our results match the classical observations of acoustic streaming in standing wave resonators. There is indeed a periodic variation in acoustic streaming velocity, which cancels at the locations of acoustic particle velocity nodes and antinodes. Moreover, it is also noticeable that the acoustic streaming flow along the centerline of the waveguide is directed toward acoustic velocity antinodes. In the case when $\phi=-\pi / 2$ (traveling wave, crosses: $\times$ ), the amplitude of the axial streaming velocity $v_{s m}\left(R_{0}, s, 0\right)$ is roughly constant and negative. This observed negative sign is in accordance with the theoretical results (see Sec. III B) which predict that, in the present case for which $\delta_{\nu} / a \approx 2 \times 10^{-3} \ll 1$, acoustic streaming is directed opposite to the traveling wave.

The spatial distribution of the outer acoustic streaming velocity with respect to the $r$-coordinate is presented in Fig. 5. Measurements are performed at position $s_{0}=0.72 \mathrm{~m}$ $\approx 3 L / 8$ and for different amplitudes of acoustic particle velocity. It clearly appears that the transverse distribution of the acoustic streaming velocity is not symmetrical with respect to the centerline. It is also noticeable that the maximum of streaming velocity is shifted toward the external wall [located at $r=R_{1}$, see Fig. 1(a)] of the resonator as the magnitude of the acoustic particle velocity increases. This behavior might be attributed to the effect of fluid inertia which leads to acoustic streaming distortion. ${ }^{14}$ In order to evaluate if the

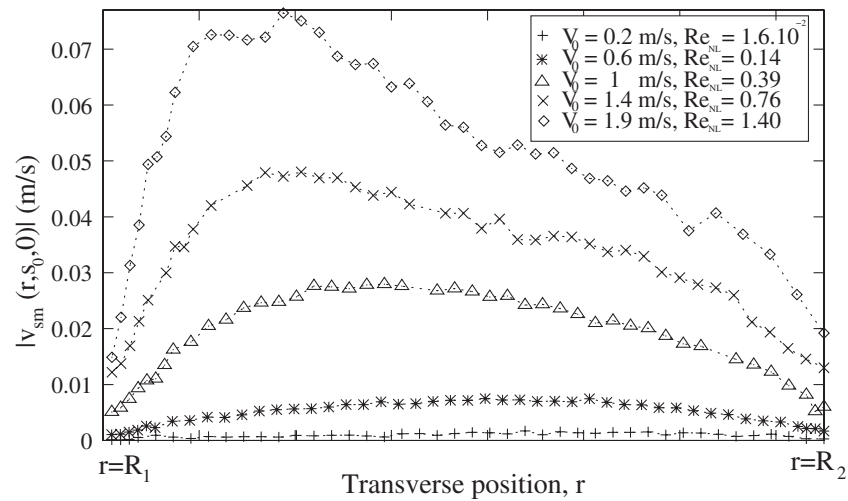

FIG. 5. Spatial distribution of the absolute values of the amplitude of the axial outer acoustic streaming velocity $\left|v_{s m}\left(r, s_{0}, 0\right)\right|$, measured by LDV at position $s_{0}=0.72 \mathrm{~m} \approx 3 L / 8$ for a phase shift $\phi=-\pi / 2$, with respect to the $r$-coordinate and for different values of the typical acoustic particle velocity $V_{0}=\left\langle v_{s}\left(r, s_{0}, 0\right)\right\rangle_{r}$.

influence of fluid inertia on acoustic streaming is important or not, a nonlinear a dimensional Reynolds number is defined as ${ }^{14}$

$$
\operatorname{Re}_{\mathrm{NL}}=\left(\frac{V_{0}}{c_{0}}\right)^{2}\left(\frac{a}{2 \delta_{\nu}}\right)^{2}
$$

where $V_{0}$ is a typical value of acoustic velocity at position $s_{0}=0.72 \mathrm{~m} \approx 3 L / 8$ (in the following, $V_{0}=\left\langle v_{s}\left(r, s_{0}, 0\right)\right\rangle_{r}$ and $\langle\cdots\rangle_{r}$ denotes taking the spatial average over the $r$-coordinate). This number $\mathrm{Re}_{\mathrm{NL}}$, which was first introduced by Menguy and Gilbert, ${ }^{14}$ is an appropriate dimensionless number which states the limit between slow and fast streaming in the kind of device considered here (i.e., frequencies in the audible range, radii of order a centimeter, acoustic levels up to $160 \mathrm{~dB}$ ). If $\mathrm{Re}_{\mathrm{NL}} \ll 1$, the corresponding streaming is called slow streaming and the effect of inertia is assumed to be negligible (so that the model presented in Appendix is valid). If $\operatorname{Re}_{\mathrm{NL}} \geq 1$, the streaming is called fast streaming (or nonlinear streaming) and fluid inertia may influence the generation of acoustic streaming, notably by distorting the shape of the transverse distribution of the acoustic streaming velocity. In the experimental results presented in Fig. 5, the parameter $\mathrm{Re}_{\mathrm{NL}}$ varies from $1.6 \times 10^{-2}$ to 1.4 so the effect of fluid inertia may be responsible for the observed asymmetry of the acoustic streaming transverse distribution. When the average amplitude of acoustic particle velocity $V_{0}$ exceeds $1 \mathrm{~m} / \mathrm{s}$ (i.e., when $\mathrm{Re}_{\mathrm{NL}} \geqslant 0.4$ ), the transverse distribution of acoustic streaming indeed becomes asymmetrical. Another plausible reason which may explain these results is that the curvature of the resonator, which impacts the transverse profile of acoustic velocity, also impacts the transverse profile of acoustic streaming velocity.

The average value $\left\langle v_{s m}\left(r, s_{0}, 0\right)\right\rangle_{r}$ over the $r$-coordinate of the acoustic streaming velocity performed at position $s_{0}$ $=0.72 \mathrm{~m} \approx 3 L / 8$ is presented in Fig. 6 as functions of $p_{\mathrm{rms}}^{2}$, where $p_{\text {rms }}$ is the root-mean-square of the acoustic pressure amplitude measured at position $s=0.75 \mathrm{~m}$. The continuous line corresponds to the linear fit of the experimental data $\left(\left\langle v_{s m}\right\rangle_{r}=\alpha_{\mathrm{fit}} p_{\mathrm{rms}}^{2}\right)$. It is verified that the acoustic streaming velocity is proportional to acoustic intensity, and the calcu- 


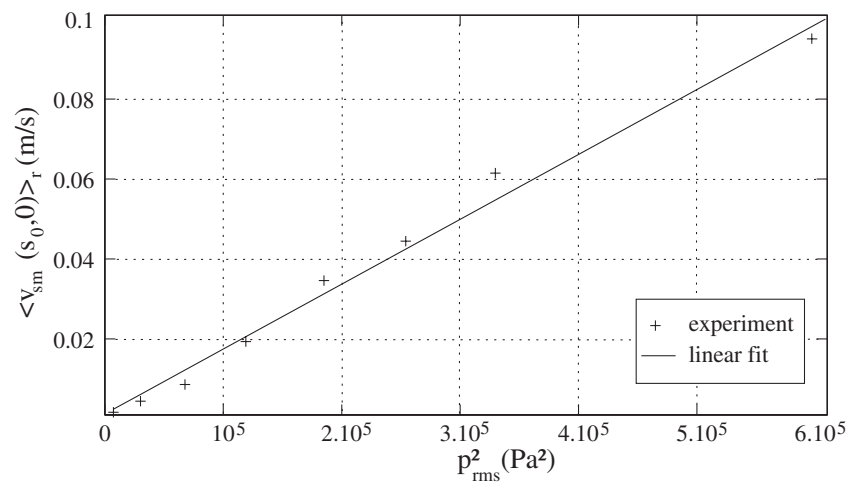

FIG. 6. Average values $\left\langle v_{s m}\left(r, s_{0}, 0\right)\right\rangle_{r}$ of the amplitude of the axial outer acoustic streaming velocity $\left|v_{s m}\left(r, s_{0}, 0\right)\right|$ over the $r$-coordinate, at position $s_{0}=0.72 \mathrm{~m} \approx 3 L / 8$, with respect to the square of the root-mean-square amplitude of the acoustic pressure $p_{\mathrm{rms}}^{2}$.

lated slope of the linear fit is $\alpha_{\mathrm{fit}} \approx-1.6 \times 10^{-7} \mathrm{~m} \mathrm{~s}^{-1} \mathrm{~Pa}^{-2}$. This value is very close to the predicted value $\alpha \approx-1.8$ $\times 10^{-7} \mathrm{~m} \mathrm{~s}^{-1} \mathrm{~Pa}^{-2}$ obtained from our simplified theory [Eq. (11)]. It is worth noting that, though the results obtained exhibit a complicated structure of acoustic streaming with a noticeable effect of fluid inertia and maybe of the curvature of the resonator, the simplified analytical predictions are not so far from experiments.

\section{CONCLUSION}

In this paper, an experimental study of a traveling wave closed-loop resonator is presented and analytical interpretations are suggested. To our knowledge, this is the first experimental study which focuses on this original device and specifically on the development of acoustic streaming generated by a resonant traveling acoustic wave. The distribution of the acoustic field, which is controlled both by the relative driving amplitude and phase shift between two drivers, is measured using LDV and the results appear to be coherent with the analytical results. The experimental analysis of the acoustic streaming development is also carried out using LDV. The obtained results show that the effects of fluid inertia and maybe the effect of the curvature of the resonator impact the spatial distribution of acoustic streaming through the cross-section of the resonator. It is also demonstrated that the measured cross-sectional average streaming velocity is in good agreement with the value predicted by our simplified theoretical model. It would be interesting in the future to repeat these experiments in the presence of a controlled temperature gradient and to compare the obtained results with analytical predictions.

\section{ACKNOWLEDGMENTS}

This work has been supported by the French National Research Agency (Contract No. ANR-05-BLANC-0016-2). The authors would like to thank Solenn Moreau, Hélène Bailliet, David Marx, and Jean-Christophe Valière for their helpful assistance and numerous advices concerning LDV measurements. The authors are also indebted to Michel Bruneau and Vitalyi Gusev for helpful discussions.

\section{APPENDIX: ESTIMATE OF THE ACOUSTIC STREAMING VELOCITY}

An order of magnitude estimate of the acoustic streaming velocity can be obtained by using the model described in Ref. 18. For the sake of simplicity, it is assumed here that the unwrapped resonator has a circular cross-section of hydraulic radius $a / 2$ (calculations would be more complicated to carry on in the case of a square cross-section). The coordinate system necessary to describe the fluid motion thus consists of an axial coordinate $y$ running along the centerline of the unwrapped resonator and a radial coordinate $r$ running perpendicular to the centerline (with $r=0$ along the centerline). The fluid motion is assumed to be symmetric about the centerline. Assuming that the boundary layer approximation is valid, the equation describing the transverse variations of the axial streaming velocity $v_{y m}$ is obtained from a successive approximations approach, ${ }^{13,18}$ leading to

$$
\begin{aligned}
\nu_{0} \frac{1}{r} \frac{\partial}{\partial r}\left(r \frac{\partial v_{y m}}{\partial r}\right)= & \frac{1}{\rho_{0}} \frac{\partial}{\partial y}\left(p_{h}+\rho_{0} \overline{v_{y}^{2}}\right)+\frac{1}{r} \frac{\partial}{\partial r}\left(r \overline{v_{y} v_{r}}\right) \\
& -\beta \frac{\nu_{0}}{T_{0}} \frac{1}{r} \frac{\partial}{\partial r}\left(r \overline{\frac{\partial v_{y}}{\partial r}}\right),
\end{aligned}
$$

where $\ldots$ is used to denote time averaging, $\tau$ denotes acoustic fluctuations of temperature, $\nu_{0}$ is the fluid viscosity evaluated at temperature $T_{0}\left(\nu_{0} \propto T_{0}^{\beta}\right.$, with $\left.\beta=0.73\right),{ }^{13} p_{h}$ is the hydrodynamic pressure accompanying the streaming, and where $v_{y}(y, r, t)$ and $v_{r}(y, r, t)$ are the axial and transverse acoustic velocities, respectively. Introducing the dimensionless coordinate $\eta=(2 r) / a$, and notifying that the crosssectional average mass flow $\langle M\rangle$ is necessarily constant $\left(\langle\cdots\rangle=2 \int_{0}^{1} \ldots \eta d \eta\right)$ due to the closed-loop geometry, it is possible to eliminate the hydrodynamic pressure $p_{h}$ in Eq. (A1) and to obtain the following expression of the acoustic streaming velocity:

$$
\left\langle v_{y m}(y)\right\rangle=\frac{1}{\rho_{0}}\left(\langle M\rangle-\left\langle\overline{\rho v_{y}}\right\rangle\right),
$$

where $\rho$ denotes acoustic fluctuations of fluid density. The cross-sectional average mass flow $\langle M\rangle$ is given by

$$
\langle M\rangle=(\oint m(y) d y) /\left(4 L \nu_{0} / a^{2}\right),
$$

where the function $m(y)$, which represents a density of sources inducing acoustic streaming, is given by

$$
\begin{aligned}
m(y)= & \left\langle\frac{\rho_{0}}{a} \int_{1}^{\eta}\left[\Re\left(\widetilde{v}_{y} \widetilde{v}_{\eta}^{*}\right)-2 \beta \frac{\nu_{0}}{T_{0}} \frac{1}{a} \mathfrak{R}\left(\widetilde{\tau}_{\frac{\partial \tilde{v}_{y}^{*}}{\partial \eta}}^{*}\right)\right] \cdot d \eta^{\prime}\right. \\
& \left.+2 \frac{\nu_{0}}{a^{2}} \mathfrak{R}\left(\tilde{\rho} \widetilde{v}_{y}^{*}\right)\right\rangle,
\end{aligned}
$$

where the relation $\overline{g h}=(1 / 2) \Re\left(\widetilde{g} \tilde{h}^{*}\right)$ (* denoting complex conjugate) is used to calculate the time average of the product $g h, \tilde{g}$ and $\tilde{h}$ being the complex amplitudes of the arbitrary functions $g$ and $h$. 
Moreover, the acoustic variables $\widetilde{\tau}, \widetilde{v}_{y}, \widetilde{v}_{\eta}$, and $\widetilde{\rho}$ can be expressed as a function of the acoustic pressure $\tilde{p}$ as follows: ${ }^{18}$

$$
\begin{aligned}
\widetilde{v}_{y}= & \frac{1-F_{\nu}}{i \omega \rho_{0}} \frac{d \widetilde{p}}{d y}, \\
\widetilde{\tau}= & \frac{1-F_{\kappa}}{\rho_{0} C_{p}} \widetilde{p}, \\
\tilde{\rho}= & \frac{1+(\gamma-1) F_{\kappa}}{c_{0}^{2}} \widetilde{p}, \\
\widetilde{v}_{\eta}= & -\frac{a}{4 i \omega \rho_{0}}\left(\frac{d}{d y}\left[\left(\eta-\Phi_{\nu}\right) \frac{d \tilde{p}}{d y}\right]\right. \\
& \left.+\left(\frac{\omega}{c_{0}}\right)^{2}\left(\eta+(\gamma-1) \Phi_{\kappa}\right) \widetilde{p}\right),
\end{aligned}
$$

where $C_{p}$ is the isobaric specific heat of fluid. In the present case of a circular cross-section, the functions $F_{\nu, \kappa}$ and $\Phi_{\nu, \kappa}$ are given by ${ }^{19}$

$$
\begin{aligned}
& F_{\kappa, \nu}=\frac{J_{0}\left(b_{\kappa, \nu} \eta\right)}{J_{0}\left(b_{\kappa, \nu}\right)}, \\
& \Phi_{\kappa, \nu}=\frac{2}{b_{\kappa, \nu}} \frac{J_{1}\left(b_{\kappa, \nu} \eta\right)}{J_{0}\left(b_{\kappa, \nu}\right)},
\end{aligned}
$$

where $b_{\kappa, \nu}=((1+i) a) /\left(2 \delta_{\kappa, \nu}\right)$, and where $J_{k}$ are the cylindrical Bessel functions of the first kind and order $k$.

As mentioned in Sec. III, it is possible to calculate the spatial distribution of acoustic pressure $\widetilde{p}(y)$ in the entire resonator, which can be reported in Eqs. (A5)-(A8) in order to calculate the average cross-sectional mass flow $\langle M\rangle$. Using Eq. (A2), the cross-sectional streaming velocity $\left\langle v_{y m}\right\rangle$ is finally obtained. This acoustic streaming velocity depends actually on the axial coordinate $y$, but in the case of a quasitraveling wave (when the displacements of the two drivers are $\pi / 2$ out of phase), it is almost constant. Indeed, after some calculations using the dimensions of the experimental device $(a=7.5 \mathrm{~cm}, L=2.12 \mathrm{~m})$, it is found that the average streaming velocity $1 / L \oint\left\langle v_{y m}\right\rangle \cdot d y$ along the closed-loop waveguide is proportional to the square of the modulus of acoustic pressure amplitude as follows:

$$
\frac{1}{L} \oint\left\langle v_{y m}\right\rangle \cdot d y \approx-\Gamma\left|\frac{1}{L} \oint \tilde{p}(y) \cdot d y\right|
$$

with $\Gamma \approx 3.6 \times 10^{-7} \mathrm{~m} \mathrm{~s}^{-1} \mathrm{~Pa}^{-2}$ and that the maximum variation of the cross-sectional average streaming velocity $\left\langle v_{y m}\right\rangle$ along the resonator is lower than $0.5 \%$ of $1 / L \oint\left\langle v_{y m}\right\rangle \cdot d y$.

${ }^{1}$ J. W. Strutt (Lord Rayleigh), "On the circulation of air observed in Kundt's tube, and on some allied acoustical problems," Philos. Trans. R. Soc. London, Ser. A 36, 10-11 (1883).

${ }^{2}$ P. Vainshtein, M. Fishman, and C. Gutfinger, "Acoustic enhancement of heat transfer between two parallel plates," Int. J. Heat Mass Transfer 38, 1893-1899 (1995).

${ }^{3} \mathrm{R}$. Moroney and R. White, "Microtransport induced by ultrasonic lamb waves," Appl. Phys. Lett. 59, 774-776 (1991).

${ }^{4} \mathrm{~N}$. Nguyen and R. White, "Design and optimization of an ultrasonic flex- ural plate wave micropump using numerical simulations," Sens. Actuators, A 77, 229-236 (1999).

${ }^{5}$ P. Luchini and F. Charru, "Acoustic streaming past a vibrating wall," Phys. Fluids 17, 122106 (2005).

${ }^{6} \mathrm{~J}$. Hu, K. Nakamura, and S. Ueha, "An analysis of a noncontact ultrasonic motor with ultrasonically levitated motor," Ultrasonics 35, 459-467 (1997).

${ }^{7}$ G. W. Swift, Thermoacoustics: A Unifying Perspective for Some Engines and Refrigerators (Acoustical Society of America, Melville, NY, 2002).

${ }^{8}$ G. Penelet, V. Gusev, P. Lotton, and M. Bruneau, "Experimental and theoretical study of processes leading to steady-state sound in annular thermoacoustic prime movers," Phys. Rev. E 72, 016625 (2005).

${ }^{9}$ G. Penelet, V. Gusev, P. Lotton, and M. Bruneau, "Non trivial influence of acoustic streaming on the efficiency of annular thermoacoustic prime movers," Phys. Lett. A 351, 268-273 (2006).

${ }^{10}$ G. W. Swift, D. Gardner, and S. Backhaus, "Acoustic recovery of lost power in pulse tube refrigerators," J. Acoust. Soc. Am. 105, 711-724 (1999).

${ }^{11}$ G. W. Swift and S. Backhaus, "A thermoacoustic Stirling heat engine," Nature (London) 399, 335-338 (1999).

${ }^{12}$ J. R. Olson and G. W. Swift, "Acoustic streaming in pulse tube refrigerators: Tapered pulse tube," Cryogenics 37, 769-776 (1997).

${ }^{13} \mathrm{~N}$. Rott, "The influence of heat conduction on acoustic streaming," ZAMP 25, 417-421 (1974).

${ }^{14} \mathrm{~L}$. Menguy and J. Gilbert, "Non-linear acoustic streaming accompanying a plane stationary wave in a guide," Acust. Acta Acust. 86, 249-259 (2000).

${ }^{15}$ M. F. Hamilton, Y. Ilinskii, and E. Zabolotskaya, "Thermal effects of acoustic streaming in standing waves," J. Acoust. Soc. Am. 114, 30923101 (2003).

${ }^{16} \mathrm{M}$. Amari, V. Gusev, and N. Joly, "Temporal dynamics of the sound wind in acoustitron," Acust. Acta Acust. 89, 1008-1024 (2003).

${ }^{17}$ D. Gedeon, "DC gas flows in Stirling and pulse-tube cryocoolers," Cryocoolers 9, 385-392 (1997)

${ }^{18}$ V. Gusev, S. Job, H. Bailliet, P. Lotton, and M. Bruneau, "Acoustic streaming in annular thermoacoustic prime-movers," J. Acoust. Soc. Am. 108, 934-945 (2000).

${ }^{19} \mathrm{H}$. Bailliet, V. Gusev, R. Raspet, and R. Hiller, "Acoustic streaming in closed thermoacoustic devices," J. Acoust. Soc. Am. 110, 1808-1821 (2001).

${ }^{20}$ M. Mironov, V. Gusev, Y. Auregan, P. Lotton, and M. Bruneau, "Acoustic streaming related to minor loss phenomenon in differentially heated elements of thermoacoustic devices," J. Acoust. Soc. Am. 112, 441-445 (2002).

${ }^{21}$ T. Biwa, Y. Tashiro, M. Ishigaki, Y. Ueda, and T. Yazaki, "Measurements of acoustic streaming in a looped-tube thermoacoustic engine with a jet pump,” J. Appl. Phys. 101, 64914 (2007).

${ }^{22} \mathrm{M}$. Thompson and A. Atchley, "Simultaneous measurement of acoustic and acoustic streaming velocities in a standing wave using laser Doppler Anemometry," J. Acoust. Soc. Am. 117, 1828-1838 (2005).

${ }^{23} \mathrm{M}$. Thompson, A. Atchley, and M. Maccarone, "Influences of a temperature gradient and fluid inertia on acoustic streaming in a standing wave," J. Acoust. Soc. Am. 117, 1839-1849 (2005).

${ }^{24} \mathrm{~S}$. Moreau, H. Bailliet, and J. C. Valière, "Measurements of inner and outer streaming vortices in a standing waveguide using laser Doppler velocimetry," J. Acoust. Soc. Am. 123, 640-647 (2008).

${ }^{25} \mathrm{~S}$. Backhaus, E. Tward and M. Petach, "Traveling-wave thermoacoustic electric generator," Appl. Phys. Lett. 85, 1085-1087 (2004).

${ }^{26} \mathrm{M}$. Amari, V. Gusev, and N. Joly, "Transient unidirectional acoustic streaming in annular resonators," Ultrasonics 42, 573-578 (2004).

${ }^{27}$ P. Ceperley, "Split mode travelling wave ring-resonator," U.S. Patent No. 4,686,407 (1987).

${ }^{28}$ C. C. Lawrenson, L. D. Lafleur, and F. D. Shields, "The solution for the propagation of sound in a toroidal waveguide with driven walls (the acoustitron)," J. Acoust. Soc. Am. 103, 1253-1260 (1998).

${ }^{29}$ P. Ceperley, "Rotating waves," Am. J. Phys. 60, 938-942 (1992).

${ }^{30}$ W. P. Arnott, H. E. Bass, and R. Raspet, "General formulation of thermoacoustics for stacks having arbitrarily shaped pore cross sections," J. Acoust. Soc. Am. 90, 3228-3237 (1991). Equation (6) is obtained by using the expression (73) in the above mentioned reference, and by assuming $\delta_{\nu, \kappa} \ll a$.

${ }^{31}$ L. Simon, O. Richoux, A. Degroot, and L. Lionet, "Laser Doppler velocimetry for joint measurements of acoustic and mean flow velocities: LMSbased algorithm and CRB calculation," IEEE Trans. Instrum. Meas. 57, 1455-1464 (2008). 